Note

\title{
A DIFFERENT SHORT PROOF OF BROOKS' THEOREM
}

\author{
LANDON RABERN \\ Arizona State University \\ School of Mathematical \& Statistical Sciences \\ e-mail: landon.rabern@gmail.com
}

\begin{abstract}
Lovász gave a short proof of Brooks' theorem by coloring greedily in a good order. We give a different short proof by reducing to the cubic case.
\end{abstract}

Keywords: coloring, clique number, maximum degree.

2010 Mathematics Subject Classification: 05C15.

\section{REFERENCES}

[1] R.L. Brooks, On colouring the nodes of a network, in: Math. Proc. Cambridge Philos. Soc. 37 Cambridge Univ. Press (1941) 194-197.

[2] R. Diestel, Graph Theory (Fourth Ed., Springer Verlag, 2010).

[3] A.D. King, Hitting all maximum cliques with a stable set using lopsided independent transversals, J. Graph Theory 67 (2011) 300-305. doi:10.1002/jgt.20532

[4] A.V. Kostochka, Degree, density, and chromatic number, Metody Diskret. Anal. 35 (1980) 45-70 (in Russian).

[5] L. Lovász, Three short proofs in graph theory, J. Combin. Theory (B) 19 (1975) 269-271. doi:10.1016/0095-8956(75)90089-1

[6] L. Rabern, On hitting all maximum cliques with an independent set, J. Graph Theory 66 (2011) 32-37. doi:10.1002/jgt.20487

[7] H. Tverberg, On Brooks' theorem and some related results, Math. Scand. 52 (1983) 37-40.

Received 28 May 2012

Accepted 3 January 2013 